Check for updates

Cite this: Mater. Adv., 2021, 2, 6039

Received 4th May 2021, Accepted 10th August 2021

DOI: 10.1039/d1ma00408e

rsc.li/materials-advances

\title{
Roles of alcohols and existing metal ions in surface chemistry and photoluminescence of InP cores $\dagger$
}

\author{
Pin-Ru Chen, Kuo-Yang Lai and Hsueh-Shih Chen (D)*
}

\begin{abstract}
Surface oxidation and chemistry determine the optical properties of indium phosphide (InP) nanocrystals (NCs). In this study, existing metal ions from the reaction residuals and alcohols normally used in the washing process are investigated. It is found that existing metal ions and alcohols play key roles in the surface oxidation and potential surface reactions of aminophosphine-based InP NCs, which significantly affect their photoluminescence (PL), optical absorption and photo-stability. According to $X$-ray photoelectron spectroscopy (XPS) analysis, InP NCs after aging form InPO $\mathrm{O}_{x}$ oxide which could passivate the NC surface along with increased PL intensity and blue-shifted spectral peaks. Treating with the existing metal ions from reaction residuals and intentionally added alcohols (methanol or ethanol), InP NCs exhibit an enhanced PL quantum yield (QY) ( 25 times, from $0.1 \%$ to $2.5 \%$ ), unexpected red-shifted spectral peaks and improved photo-stability ( $\sim 9$ folds). The phenomenon is ascribed to the passivation effect of the $Z n_{y} \mid n_{1-y} P_{x}$ layer formed from coupling of existing $\mathrm{Zn}^{2+}$ with the $\mathrm{NC}$ surface triggered by the alcohols. The alcohols are found to facilitate the surface modification of amine-capped NCs and may be utilized as agents in NC surface modifications.
\end{abstract}

\section{Introduction}

Colloidal nanocrystals (NCs) have attracted considerable attention owing to their shape- and size-dependent optical and electronic properties. ${ }^{1,2}$ The intriguing properties make NCs promising building blocks in a variety of applications such as light emitting diodes (LEDs),,$^{3-7}$ biolabeling, ${ }^{8,9}$ lasers $^{10,11}$ and solar cells. ${ }^{12,13}$ However, concerns over toxic metals such as $\mathrm{Pb}$ - and Cd-based NCs restrict their practical applications. Indium phosphide (InP) NCs with nontoxicity, size-tuneable bandgap in the entire visible range and good optical properties are considered as a promising alternative material of toxic NCs. ${ }^{14-16}$ Unfortunately, synthesis of high-quality InP NCs is still challenging because of the covalent nature for III-V semiconductors, native oxides or surface dangling bonds on InPbased NCs. ${ }^{17-19}$

Aminophosphine, including tris-dimethylaminophosphine $\left((\mathrm{DMA})_{3} \mathrm{P}\right)$ and tris-diethylaminophoshpine $\left((\mathrm{DEA})_{3} \mathrm{P}\right)$, as an alternative $\mathrm{P}$ precursor to synthesize high-quality InP NCs, is a safer and more inexpensive choice compared with tris(trimethylsilyl)phosphine $\left((\mathrm{TMS})_{3} \mathrm{P}\right) \cdot{ }^{20-22}$ However, synthetic protocols of InP NCs are still complicated since InP NCs are

Quantum Dots and Green Materials Lab, Department of Materials Science and Engineering, National Tsing Hua University, Hsinchu 30013, Taiwan.

E-mail: chenhs@mx.nthu.edu.tw

$\dagger$ Electronic supplementary information (ESI) available. See DOI: 10.1039/d1ma00408e extremely susceptible to surface oxidation that may affect subsequent shell growth or surface modification. ${ }^{23-25}$

Accordingly, a precise control over the NC surface is considered a crucial factor to prepare high-quality NCs. As well as water, which is already regarded as a critical factor to oxidation, ${ }^{26-28}$ the NC surface with surrounding environments is another factor that strongly affects the surface chemistry of NCs and their resulting properties. For example, several studies have investigated the relation between surface chemistry, oxidation and stability of NCs under varied environments such as atmospheres, irradiation, temperatures and additives either in solutions or thin films. ${ }^{29-36}$

The surface chemistry of InP NCs is dependent on their surface ligands, that may be acid-capped or amine-capped InP NCs. Surface oxidation study of acid-capped InP NCs prepared from toxic (TMS $)_{3} \mathrm{P}$ have been reported. ${ }^{37-40}$ For eco-friendly aminophosphine-based InP NCs, the surface chemistry and oxidation mechanism still remain unclear. ${ }^{24,41}$ In this work, the roles of existing metal ions and intentionally added alcohols on aminophosphine-based InP NC oxidation and surface chemistry are investigated. In a general aging process without additives, the aged InP NCs show an increased $\mathrm{InPO}_{x}$ oxide layer with enhanced photoluminescence (PL) intensity and blue-shifted spectral peaks. However, aging or treating InP NCs involving reaction residuals (i.e., $\mathrm{In}^{3+}, \mathrm{Zn}^{2+}, \mathrm{Cl}^{-}$and oleylamine (OAm)) with intentionally added alcohols results 
in enhanced PL and unexpected redshifted spectral peaks. X-ray photoelectron spectroscopy (XPS) proves that alcohol induces incorporation of $\mathrm{Zn}^{2+}$ to form a mixed $\mathrm{Zn}_{y} \mathrm{In}_{1-y} \mathrm{PO}_{x}$ oxide passivation layer. The $\mathrm{Zn}_{y} \mathrm{In}_{1-y} \mathrm{PO}_{x}$ renders enhancement not only in PLQY but also photo-stability of InP NCs against aging. The reaction between alcohol and $\mathrm{Zn}^{2+}$ creates a beneficial effect on aminophosphinebased InP NCs. This study may provide a new insight to surface chemistry, following surface modification and overcoating for aminophosphine-based InP NCs.

\section{Experimental}

\subsection{Chemicals}

Indium chloride $\left(\mathrm{InCl}_{3}, 99.999 \%\right)$ and oleylamine (OAm, technical grade, 70\%) were purchased from Sigma-Aldrich. Zinc chloride $\left(\mathrm{ZnCl}_{2}\right.$, anhydrous, 99.95\%) and tris-dimethylaminophosphine $\left((\mathrm{DMA})_{3} \mathrm{P}, 97 \%\right)$ were purchased from Alfa Aesar. All solvents were purchased from J. T. Baker.

\subsection{Synthesis of aminophosphine-based InP NCs}

Aminophosphine-based InP NCs were prepared by a modified method reported by Tessier et al. ${ }^{21}$ Briefly, $100 \mathrm{mg}$ of $\mathrm{InCl}_{3}$ and $300 \mathrm{mg}$ of $\mathrm{ZnCl}_{2}$ were mixed with $5 \mathrm{~mL}$ technical OAm in a three neck flask inside a $\mathrm{N}_{2}$-filled glovebox then transferred to a Schelenk line. The mixture was degassed at $120{ }^{\circ} \mathrm{C}$ under vacuum conditions for $1 \mathrm{~h}$ and then heated to an elevated temperature under an inert atmosphere. At the reaction temperature $\left(150-280{ }^{\circ} \mathrm{C}\right), 0.45 \mathrm{~mL}$ of $(\mathrm{DMA})_{3} \mathrm{P}$ was injected into the reaction mixture, and the InP core growth was lasted for $20 \mathrm{~min}$. After the reaction, the crude solution was cooled to room temperature, and InP NCs were directly collected from the reaction mixture for subsequent aging processes. The washing process was presented as follows. $50 \mu \mathrm{L}$ of InP NCs were mixed with $450 \mu \mathrm{L}$ toluene and were precipitated by adding $1 \mathrm{~mL}$ acetone. The precipitates were re-dispersed in toluene and quantified to a fixed concentration for subsequent treatment and analyses.

\subsection{Sample preparation for the aging process}

InP NCs were quantified by comparing the absorbance of quantitative aliquots at $413 \mathrm{~nm}$ with the intrinsic absorption coefficient of InP NCs at the same wavelength. ${ }^{21}$ In the aging processes, the samples were prepared by diluting InP NCs with toluene, and the concentration of InP NCs was fixed at $1 \mathrm{mM}$ with a total amount of $3 \mathrm{~mL}$ for all experiments. The washed InP (W-InP) samples were prepared by a washing process using acetone to remove reaction residuals. The concentration of W-InP samples was also quantified to $1 \mathrm{mM}$. An Ar atmosphere was achieved by sealing the sample and flooding in $\mathrm{Ar}$ for $10 \mathrm{~min}$ prior to experiments. An air atmosphere was prepared by removing the lid of the sample and placing it under air for 10 min followed by sealing the sample. The dark samples were achieved by filling the sample with the corresponding atmosphere and wrapping the sample with aluminium foil. The light samples were irradiated by a white light tube with illuminance of 500 lux. All additives (i.e., amines, thiols, alcohols, 1-octadecene (ODE), acetone, oleic acid (OA) and $\mathrm{H}_{2} \mathrm{O}_{2}$ ) were added in an amount of $1 \mathrm{mmol}$ in the corresponding samples prior to the aging process.

\subsection{Alcohol treatment}

In brief, $50 \mu \mathrm{L}$ of InP NCs were mixed with $450 \mu \mathrm{L}$ toluene and $1 \mathrm{~mL}$ ethanol or methanol followed by centrifugation. The precipitates were collected and re-dispersed in toluene for further analyses. The ethylene glycol (EG) treatment was conducted by replacing methanol with EG. Regarding the immiscibility of EG and toluene, rather than centrifugation, the supernatant of InP NCs in toluene were collected and analysed after the treatment. A control sample was prepared by replacing methanol with acetone having no additional effect on InP NCs while the other procedures were remained the same.

\subsection{Characterization}

Optical absorption spectra were recorded with a UV-vis spectrometer (HITACHI U-3900) in $1 \mathrm{~cm}$ path length quartz cuvettes. The PL spectra of InP NCs dispersed in toluene were recorded using a PL spectrometer (HORIBA FluoroMax-4). The PL quantum yield (QY) was determined by comparing InP NC fluorescence intensities with those of primary standard dye solution (Rhodamine 6G, QY $=95 \%$ in ethanol) with the same optical density and the excitation wavelength. X-ray photoelectron spectroscopy (XPS) was employed to determine the chemical states of elements in InP NCs. The XPS spectra were measured by Ulvac-PHI, PHI 5000 VersaProbe II. The binding energy of the XPS spectra was calibrated from a hydrocarbon using the $\mathrm{C}$ 1s peak at $284.6 \mathrm{eV}$. Estimation of the chemical composition from XPS analysis was calculated based on Scofield's relative sensitivity factors (RSF). Thermogravimetric analysis (TGA) curves were recorded by a Q500 thermogravimetric analyser under a $\mathrm{N}_{2}$ atmosphere using a heating rate of $10{ }^{\circ} \mathrm{C} \mathrm{min}^{-1}$ starting from room temperature to $700{ }^{\circ} \mathrm{C}$. During the measurement, the temperature was maintained at $100{ }^{\circ} \mathrm{C}$ for $30 \mathrm{~min}$ to ensure evaporation of any trapped solvent. The samples were prepared by dropping the QD solution into aluminium oxide crucibles. Time-resolved PL (TRPL) spectra were obtained from PicoHarp300 with TTTR Mode and PHR 800 router (PicoQuant).

\section{Results and discussion}

\subsection{Evolution of the oxidation of InP NCs in an aging process}

Aminophosphine-based InP NCs are prepared from (DMA) ${ }_{3} \mathrm{P}$, $\mathrm{InCl}_{3}, \mathrm{ZnCl}_{2}$ and OAm at an elevated temperature. The asprepared InP NCs directly collected from the reaction mixture show the first excitonic absorption and band-edge PL emission at 570 and $628 \mathrm{~nm}$, respectively (Fig. S1, ESI $\dagger$ ). The PLQY is rather low $(\sim 0.1 \%)$ and has been generally attributed to the surface defect states of InP NCs. ${ }^{42,43}$ A defect-related PL emission is also observed at $\sim 750 \mathrm{~nm}$. III-V InP NCs are prone to oxidation, and the oxidation affects the InP optical properties to a large extent. ${ }^{44}$ The InP NCs directly collected from the 
a

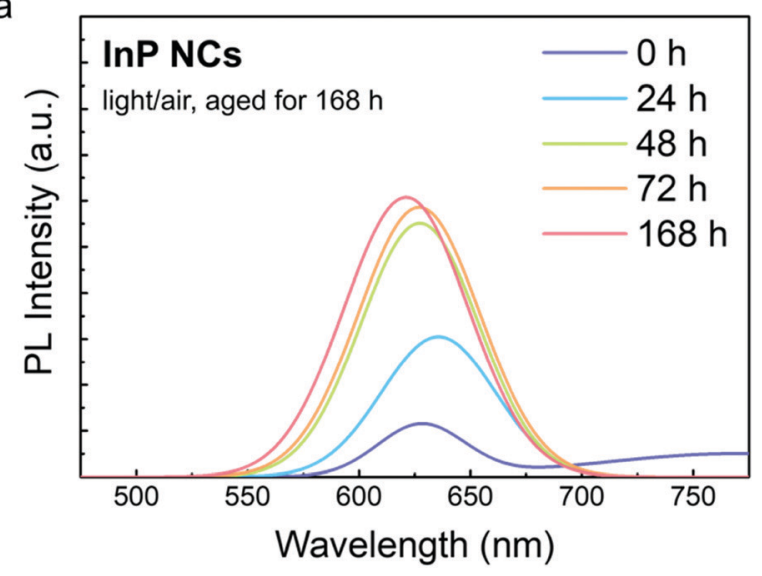

$\mathrm{C}$

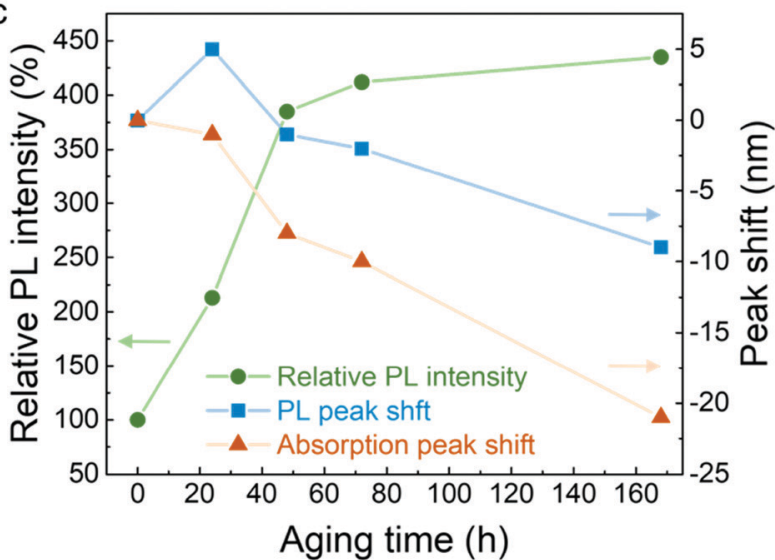

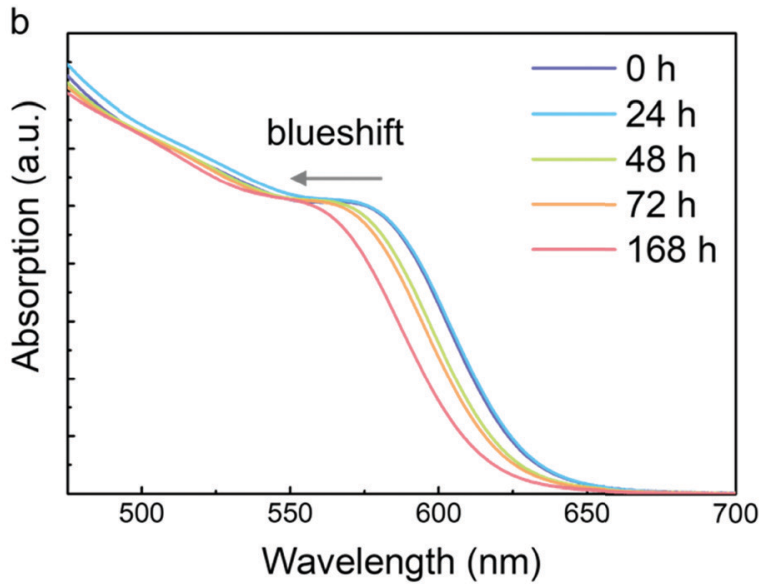

d

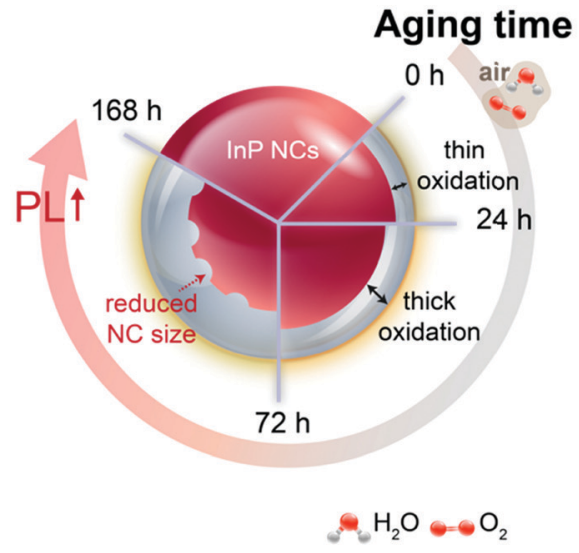

Fig. 1 Optical properties of InP NCs during aging under light/air conditions for $168 \mathrm{~h}$. The sample is in a concentration of $1 \mathrm{mM}$ with a total amount of $3 \mathrm{~mL}$ in toluene. Evolution of (a) PL and (b) optical absorption spectra of InP NCs during aging. (c) Variation of relative PL intensity, PL peak shift and absorption peak shift against aging time. (d) A sketch illustrates the evolution of InP NC oxidation in the aging process.

reaction mixture are without a washing process, and the effect of surface ligands and reaction residuals (i.e., $\mathrm{In}^{3+}, \mathrm{Zn}^{2+}, \mathrm{Cl}^{-}$, $\mathrm{P}(\mathrm{NHR})_{4}{ }^{+}$and $\left.\mathrm{OAm}\right)$ may be magnified.

Ex situ PL study results of InP NCs aged under room light in an atmosphere (light/air) for $168 \mathrm{~h}$ are shown in Fig. 1a. The InP NCs show an increased PL intensity to $213 \%, 385 \%$ and $435 \%$ after aging for $24 \mathrm{~h}, 48 \mathrm{~h}$ and $168 \mathrm{~h}$, respectively. It is noted that the PL peak redshifts to $633 \mathrm{~nm}$ in $24 \mathrm{~h}$, and blueshifts back to $627 \mathrm{~nm}$ in $48 \mathrm{~h}$, leading to an overall $9 \mathrm{~nm}$ PL blueshift in $168 \mathrm{~h}$. Unlike the PL spectra, the corresponding optical absorption peak slightly blueshifts $2 \mathrm{~nm}$ in $24 \mathrm{~h}$ and constantly blueshifts for $21 \mathrm{~nm}$ after $168 \mathrm{~h}$ aging (Fig. 1b). Variations of PL intensity, PL peak shift and optical absorption peak shift are shown in Fig. 1c and summarized in Table S1 $(\mathrm{ESI} \dagger)$.

In the first $24 \mathrm{~h}$ aging, the slight blueshift of the absorption peak indicates a decrease in the InP NC size, which is ascribed to oxidation at the InP NC near-surface region..$^{4-47}$ The thin oxidation layer could passivate the non-radiative defects such as surface dangling bonds and thus increase the PL intensity. Meanwhile, the oxidation layer might also create additional radiative states, acting as luminescent centres lying within the bandgap. The photo-excited carriers could delocalize to the lower energy radiative states, and a slight PL redshift is observed. $^{48,49}$

As the aging and oxidation process continue, the further blue-shifted optical absorption suggests further decrease in InP NC size and a thicker surface oxidation layer, which might lead to further increase in PL intensity. A similar result that described PL enhancement of InP NCs after air exposure was reported previously. ${ }^{45}$ Illustration of InP NC oxidation in the aging process is schematically shown in Fig. 1 d.

It has been reported that the effect of NC oxidation may be either positive or negative on the optical properties of NCs (e.g., PL intensity). ${ }^{29,39}$ As stated above, formation of a surface oxide layer over the NC surface could not only passivate the surface defects but also create structural inconsistency with additional defects. ${ }^{29}$ Relatively low PLQY $(<1 \%)$ of the InP NCs suggests large amounts of nonradiative defects at the NC surface. ${ }^{23}$ Thus, the oxidation layer can improve the defective InP NC surface with increased PL intensity in the current study.

\subsection{Effect of reaction residuals on the InP NC oxidation}

To investigate the effect of the reaction residuals on InP NC oxidation, washed InP (W-InP) NCs are utilized for comparison. W-InP NCs are prepared by centrifugation with acetone to 
remove more reaction residuals. Typically, InP and W-InP NCs have identical PL and absorption properties, the only difference is the amount of reaction residuals. Reaction residuals may contain $\mathrm{In}^{3+}, \mathrm{Zn}^{2+}, \mathrm{Cl}^{-}, \mathrm{P}(\mathrm{NHR})_{4}{ }^{+}$and $\mathrm{OAm}$, and their deduced concentrations are listed in Table S2 (ESI $\dagger$ ). The InP/reaction residuals weight ratio of InP and $\mathrm{W}$-InP NCs are approximately $1 / 70$ and $1 / 1.2$, respectively. W-InP NCs possess the cubic structure and the diameter of $2.6 \pm 0.4 \mathrm{~nm}$ according to XRD and TEM analyses (Fig. S3, ESI $\dagger$ ). A detailed estimation method and more material properties of InP and W-InP NCs are provided in the ESI. $\dagger$

The effects of different aging conditions, including room light in $\mathrm{Ar}$ (light/Ar), room light in the atmosphere (light/air), dark in $\mathrm{Ar}$ (dark/Ar) and dark in the atmosphere (dark/air) with an aging time of $48 \mathrm{~h}$, on the optical properties of both InP NCs are investigated. Detailed experimental procedures which involve the light source, preparation of dark conditions and different atmospheres are described in the Experimental section. As shown in Fig. 2a, aged InP NC samples in light/Ar, light/air, dark/Ar and dark/air show enhanced PL intensities of $260 \%, 392 \%, 191 \%$ and $222 \%$, respectively. Meanwhile, the aged W-InP NC samples in light/Ar, light/air, dark/Ar and dark/ air exhibit the PL intensity of $187 \%, 40 \%, 169 \%$ and $171 \%$, respectively. As shown in Fig. 2b, all aged InP and aged W-InP NC samples show blue-shifted optical absorption spectra, in which aged W-InP samples exhibit larger blueshifts compared with the aged InP NC counterparts.

Exposure to light generally accelerates oxidation of NCs via a photo-oxidation process. When semiconductor NCs are illuminated by light, photo-excited carriers could delocalize to the NC surface and interact with surrounded species such as oxygen and water, then the surface oxidation process is accelerated. ${ }^{29,50}$ As stated above, a thicker oxidation layer may occur with a largely blueshifted absorption curve and increased PL intensity. The InP samples aged with light illumination exhibit larger spectral blueshift and stronger PL enhancement than those aged in the dark. On the other hand, InP samples aged in the atmosphere possess higher PL intensities than those aged in Ar, suggesting that components in the atmosphere might also accelerate surface oxidation.

Compared with the abovementioned InP NCs, W-InP NCs after aging in various conditions all show lower PL intensities. Notably, the aged W-InP light/air sample shows a significant decrease in the PL intensity $\sim 60 \%$ along with a large absorption blueshift $\sim 50 \mathrm{~nm}$, while the other three W-InP samples aged in relatively "gentle" environments (light/Ar, dark/Ar and dark/air) still show increased PL intensities. A large absorption blueshift in the aged W-InP light/air sample implies a large decrease in the NC size because of more serious surface oxidation, which could generate more non-radiative defects attributed to a decrease in PL intensity.

Based on the above results, W-InP NCs with fewer reaction residuals may be more easily attacked by oxygen and water in the aging process. Strong oxidation with downgraded optical properties of aged W-InP NCs are observed. On the contrary, InP NCs with more reaction residuals, which prevent NCs from direct contact with oxygen and water, show improved PL intensity after the aging process.

In previous studies, when Cd-based NCs were continuously illuminated by light, initially increased PL intensities followed by constantly decreased PL intensities were observed. The phenomenon is generally called photo-brightening and photodarkening, respectively. ${ }^{29,33,51-53}$ It should be noted that the PL enhancement in the current study is different from the reported photo-brightening and photo-darkening.

In the current study, the PL enhancement still occurs for InP NCs in dark conditions, that may be oxidized after aging because of the highly sensitive nature of III-V semiconductor materials. ${ }^{37,54}$ Exposure to light would simply accelerate the NC oxidation process. A sketch illustrating the effect of aging condition on InP NC oxidation is shown in Fig. 2c. A summary
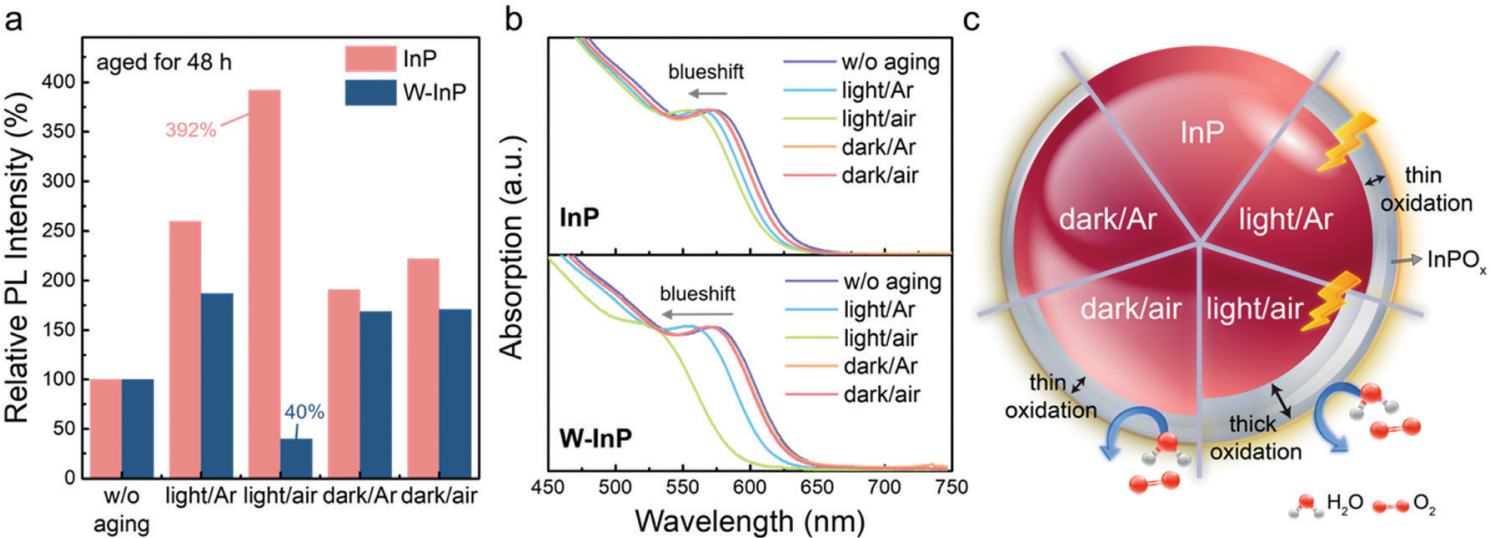

Fig. $2 \ln \mathrm{P}$ and washed $\operatorname{InP}(\mathrm{W}-\mathrm{InP}) \mathrm{NCs}$ aged under different aging conditions for $48 \mathrm{~h}$. The concentration of all samples is fixed at $1 \mathrm{mM}$ with a total amount of $3 \mathrm{~mL}$ in toluene. The $\mathrm{W}$ - InP sample is prepared from $\mathrm{InP} N C$ s by centrifugation to remove reaction residuals/excess ligands using acetone. Detailed experimental procedures including the light source, preparation of dark conditions and different atmospheres are described in the Experimental section. (a) Relative PL intensity of InP and W-InP NCs aged under light/Ar, light/air, dark/Ar and dark/air for 48 h. (b) Optical absorption spectra of the InP and W-InP NCs after the corresponding aging process. (c) A sketch illustrates the effect of atmosphere and light illumination on InP NC oxidation in the aging process. 
of optical characteristics of both aged InP and aged W-InP NCs is shown in Table S4 (ESI $\dagger$ ).

XPS surface analysis of InP NCs and aged InP NCs is presented in Fig. 3 and Fig. S4 (ESI $\dagger$ ). Fig. 3a shows the XPS In 3d spectra of the InP NC sample without aging for comparison, and the aged InP NCs in light/air for $48 \mathrm{~h}$ and aged $\mathrm{W}$-InP NCs in light/air for $48 \mathrm{~h}$. The In $3 \mathrm{~d}$ doublet peaks of the InP NC sample without aging (top spectrum) show a spin-orbit splitting of $7.6 \mathrm{eV}$ with $3 \mathrm{~d}_{5 / 2}$ and $3 \mathrm{~d}_{3 / 2}$ peaks at 444.2 and $451.8 \mathrm{eV}$, respectively. The doublet is deconvoluted into two subdoublets. The light-red curve (doublet at 444.1 and $451.7 \mathrm{eV}$ ) is ascribed to the In-P binding, while the dark-red one (doublet at 444.9 and $452.5 \mathrm{eV}$ ) is assigned to $\mathrm{In}-\mathrm{PO}_{x}$ from the oxidized InP NCs. ${ }^{55,56}$ The In- $\mathrm{PO}_{x} / \mathrm{In}-\mathrm{P}$ peak intensity ratio is 0.3 . For the aged InP (middle spectrum) and aged W-InP (bottom spectrum) NC samples, the In- $\mathrm{PO}_{x} / \mathrm{In}-\mathrm{P}$ peak intensity ratios increase to 0.9 and 1.7 , respectively.

As shown in Fig. 3b, similar results are observed in the XPS P 2p spectra. The InP sample shows a peak at $128.4 \mathrm{eV}$ ascribed to the $\mathrm{P}-$ In (light-blue doublet) and a peak at $132.6 \mathrm{eV}$ assigned to $\mathrm{P}-\mathrm{O}_{x}$ (InPO ${ }_{x}$, dark-blue doublet) with a spin-orbit splitting of $0.87 \mathrm{eV}$ between $2 \mathrm{p}_{1 / 2}$ and $2 \mathrm{p}_{3 / 2} \cdot{ }^{47,48}$ The $\mathrm{InPO}_{x} / \mathrm{InP}$ intensity ratios are 0.3 , 0.9 and 1.6 for the InP, aged InP and aged W-InP samples, respectively. Calculated $\mathrm{InPO}_{x} / \mathrm{InP}$ ratios from the $\mathrm{P} 2 \mathrm{p}$ spectra have a similar trend with that from the In $3 \mathrm{~d}$ spectra. Fig. $3 \mathrm{c}$ gives the atomic ratio of oxygen/phosphorus $(\mathrm{O} / \mathrm{P})$, carbon/phosphorus $(\mathrm{C} / \mathrm{P})$ and zinc/phosphorus $(\mathrm{Zn} / \mathrm{P})$ calculated from the XPS spectra. The aged $\mathrm{W}$-InP sample shows a relatively larger $\mathrm{O} / \mathrm{P}(\sim 4.9)$ and smaller $\mathrm{C} / \mathrm{P}(\sim 9.3)$ ratio compared with the aged InP sample. All samples present a slight $\mathrm{Zn}$ content (ratio of $\mathrm{Zn} / \mathrm{P} \sim 0.1$ ) ascribed to the surface adsorbed $\mathrm{Zn}^{2+}$ of InP NCs originally from the reaction residuals. The elemental ratios of indium/phosphorus (In/P) and nitrogen/phosphorus (N/P) are similar in all InP NC samples (Fig. S5, ESI $\dagger$ ).

Based on the above results, it is suggested that InP NCs have native $\mathrm{InPO}_{x}$ that may inevitably form in the crystal growth process because of trace water in the synthetic reaction. ${ }^{38}$ After the light/air aging process, more $\mathrm{InPO}_{x}$ would form at the NC surface. Considering the above PL properties with the extent of oxidation, the $\mathrm{InPO}_{x}$ oxidation formed in the aging process would first improve the PL intensity with a blue-shifted absorption peak $(\sim 10 \mathrm{~nm})$, as shown in the aged InP sample. When the oxidation becomes more serious, the $\mathrm{InPO}_{x}$ oxide layer would cause a decrease in the PL intensity and a large blueshift in absorption peak $(\sim 50 \mathrm{~nm})$, as shown in the aged $\mathrm{W}$-InP sample. The strong oxidation is also supported by the decreased $\mathrm{C} / \mathrm{P}$ ratio indicating ligand desorption on heavily oxidized NCs. Compared the aged InP with aged W-InP samples, the reaction residuals in the sample indeed mitigate surface oxidation in the aging process. It is suggested that reaction residuals are beneficial to prevent serious oxidation and render a proper surface passivation of InP NCs. The similar $\mathrm{Zn} / \mathrm{P}, \mathrm{In} / \mathrm{P}$ and N/P ratios of InP, aged InP and aged $\mathrm{W}$-InP NCs suggest the reaction residuals would not be involved in the InPO ${ }_{x}$ oxide layer in the aging process.

The passivation effect of the $\mathrm{InPO}_{x}$ oxide layer is also supported by time-resolved PL (TRPL) spectra (Fig. S6 and Table S5, ESI $\dagger$ ). An increase in the PL lifetime of the aged InP sample indicates better surface passivation by the $\operatorname{InPO}_{x}$ oxide layer along with increased PL intensity. The increased lifetime of the long-lifetime component related to the surface defect states of the aged W-InP NC sample suggests serious oxidation with a rather thick and defective $\mathrm{InPO}_{x}$ layer on the aged $\mathrm{W}$-InP NCs. Attempts have been made to investigate InP NC oxidation by TEM analysis, however, small size and low $Z$-contrast of InP NCs make the observation infeasible.

\subsection{Effect of additives on InP NC PL enhancement and an alcohol-induced passivation}

Despite the reaction residuals, the effect of additives on InP NC oxidation and surface chemistry are investigated. Several additives (i.e., amines, thiols, alcohols, 1-octadecene (ODE), acetone, oleic

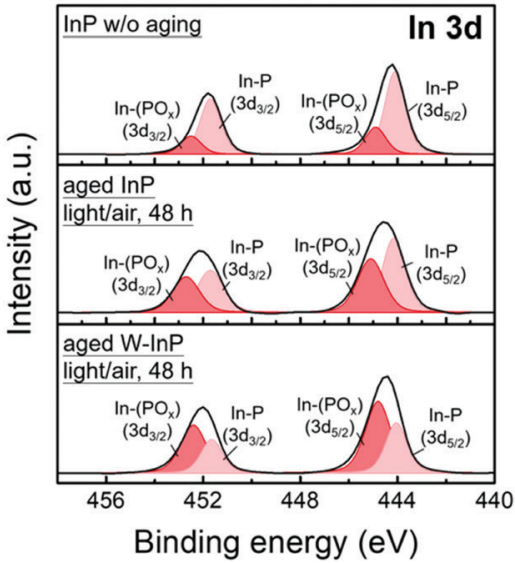

b

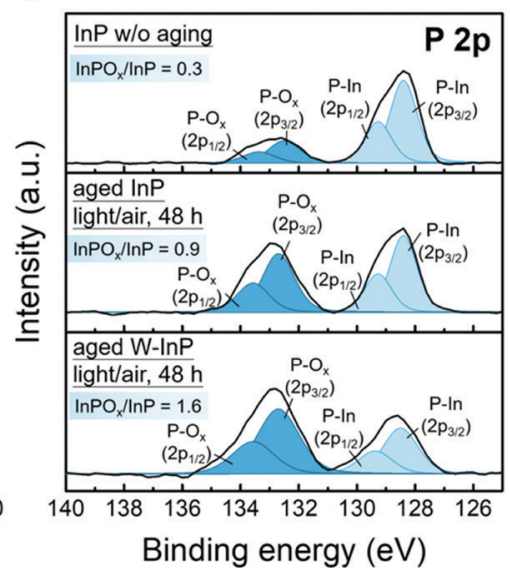

C

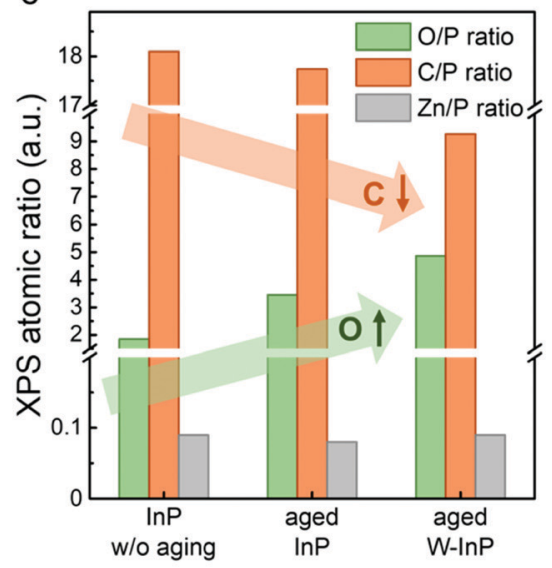

Fig. 3 XPS (a) In 3d, (b) P 2p spectra and (c) calculated relative oxygen/phosphorus (O/P), carbon/phosphorus (C/P) and zinc/phosphorus (Zn/P) atomic ratio of the $\operatorname{InP}$, aged $\operatorname{InP}$ and aged $\mathrm{W}$ - InP NC samples. InP NC sample without aging is used for comparison. The concentration of the aged samples is fixed at $1 \mathrm{mM}$ with a total amount of $3 \mathrm{~mL}$ in toluene with an aging process in light/air for $48 \mathrm{~h}$. 
a

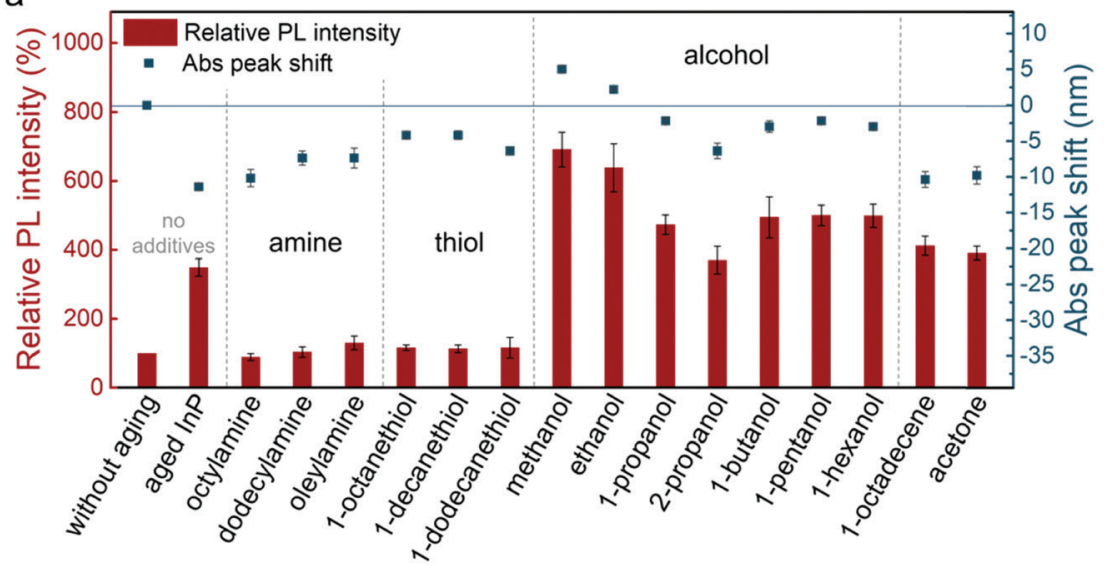

b

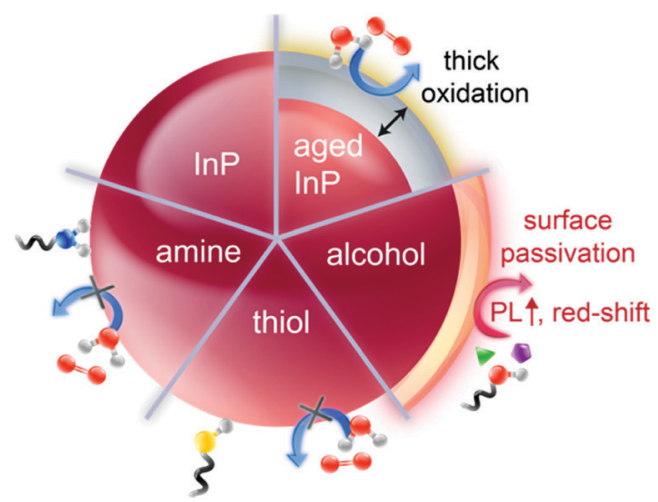

Fig. 4 Effect of additives on PL and optical absorption of InP NCs aging in light/air for $48 \mathrm{~h}$. The concentration of all samples is fixed at $1 \mathrm{mM}$ with a total amount of $3 \mathrm{~mL}$ in toluene. All additives are added in an amount of $1 \mathrm{mmol}$. (a) Relative PL intensity and absorption peak shift of InP NCs with various additives, i.e., amines (octylamine, dodecylamine and oleylamine), thiols (1-octanethiol, 1-decanethiol and 1-dodecanethiol), alcohols (methanol, ethanol, 1-propanol, 2-propanol, 1-butanol, 1-pentanol and 1-hexanol), 1-octadecene (ODE) and acetone. (b) A sketch illustrates the effect of amines, thiols and alcohols on InP NC oxidation in the aging process.

acid (OA) and $\mathrm{H}_{2} \mathrm{O}_{2}$, ) are intentionally added in the InP NC samples, and the samples are aged in light/air for $48 \mathrm{~h}$. Variation of PL intensity and optical absorption peak shift of the samples are shown in Fig. 4a. Optical absorption spectra of the samples are presented in Fig. S7 (ESI $\dagger$ ). An aged InP NC sample without any additive is listed for comparison. As mentioned, the aged InP sample shows an absorption peak blueshift of $11 \mathrm{~nm}$ and enhanced PL intensity $\sim 350 \%$ caused by $\operatorname{InPO}_{x}$ oxide passivation. If amine or thiol is introduced in the aging process, amine-additive and thiol-additive samples show no enhancement in PL intensity but a slightly blueshifted absorption peak (5-10 nm). Interestingly, alcohol-additive samples show a substantial increase in PL intensities, especially for methanol and ethanol-additive samples. The methanol-additive sample shows the highest PL intensity up to $691 \%$ while the other alcohol-additive samples exhibit PL intensities in a range of 370$638 \%$. Only small absorption peak-shifts (blueshift for 2-5 nm) are observed in alcohol-additive samples, and even a redshift of 5 and $2 \mathrm{~nm}$ for the methanol-additive and ethanol-additive samples, respectively. Moreover, when non-coordinating ODE and acetone are used as the additive, both samples show similar properties (PL intensity $\sim 350 \%$ and absorption blueshift $\sim 10 \mathrm{~nm}$ ) as the aged InP NC sample without any additive. In terms of OA- and $\mathrm{H}_{2} \mathrm{O}_{2}$ additive, some significant changes after the aging process can be directly observed by the naked eye. As a photo demonstrating the samples after aging in Fig. S8 (ESI $\dagger$ ), the InP NC sample after aging still has a light-orange colour while the OA- and $\mathrm{H}_{2} \mathrm{O}_{2}$ additive samples become transparent and white-turbid solutions, respectively.

These results suggest that the additives could indeed affect InP NC oxidation and optical properties in the aging process. The added amines and thiols may readily interact with InP NCs since they are common ligands for stabilizing NCs. ${ }^{57,58}$ Regarding the large increase in PL intensity and redshift in the absorption spectra of the methanol-additive sample, formation of the $\mathrm{InPO}_{x}$ oxide layer may not be the only cause for this phenomenon. As stated above, the InP NCs aged without additives show increased InPO$_{x}$ with enhancement in PL intensity and blueshift in the absorption peak. Therefore, the alcohols are suggested to bind to InP NCs or react with InP NCs and/or reaction residuals for such different results. In addition, the ODE-additive and acetone-additive samples show similar changes as the InP NC sample without any additives. The results imply both ODE and acetone have no additional effect on InP NC oxidation or PL enhancement. In other words, amines, thiols and alcohols do either bind or react with NCs for unchanged PL or substantially increased PL after the aging 
process. The visible changes in $\mathrm{OA}$ - and $\mathrm{H}_{2} \mathrm{O}_{2}$-additive sample solutions may be associated with the etching effect from OA and over oxidation from $\mathrm{H}_{2} \mathrm{O}_{2}$ as the oxidative agent, ${ }^{55,56}$ highlighting the vulnerability of InP NCs. The effect of amines, thiols and alcohols on InP NC oxidation and PL properties in the aging process is illustrated in Fig. $4 \mathrm{~b}$.

\subsection{Formation of the $\mathrm{Zn}_{y} \operatorname{In}_{1-y} \mathrm{PO}_{x}$ passivation layer induced by alcohol}

To clarify the effect of alcohols on the InP NC oxidation and PL properties, the relation of alcohol polarity with the corresponding PL intensity of the aged alcohol-additive sample is shown in Fig. 5a. ${ }^{59}$ The higher PL intensity occurs along with higher polarity alcohol. Thus, methanol and ethanol, exhibiting the two highest PL intensities after the aging process, are used to directly treat InP NCs to examine the underlying mechanism. InP NCs directly collected from the reaction mixture are diluted with toluene to a fixed concentration (InP/toluene). The InP/ toluene solution is then mixed with methanol or ethanol, and the mixture undergoes a centrifugation process, which is denoted as the post alcohol treatment in this study. A control sample is prepared by treating InP/toluene with acetone exhibiting no additional effect on InP NC oxidation in the above aging process. Optical absorption and PL spectra of the alcoholtreated samples are shown in Fig. 5b. The control sample shows an absorption peak at $570 \mathrm{~nm}$ with a low PLQY $\sim 0.1 \%$. After post alcohol treatment, the methanol-treated InP (MeOH-InP) sample shows an apparently increased PLQY of $2.4 \%$ with a large absorption peak redshift of $12 \mathrm{~nm}$ while the ethanoltreated InP (EtOH-InP) sample exhibits a PLQY of 1.2\% and an absorption peak redshift of $7 \mathrm{~nm}$. Increases in peak-to-valley ratios in absorption curves (inset in Fig. 5b) are also observed after the post alcohol treatment. Another alcohol, ethylene glycol (EG, relative polarity of water $=0.790)$, with two hydroxyl groups and also a high polarity ${ }^{59}$ is also tested in the alcohol treatment. Due to the immiscibility of EG with InP/toluene, rather than centrifugation, the sample is prepared by simply stirring EG with InP/toluene, and the supernatant InP/toluene is taken out for analyses. The EG-treated InP NCs similarly show an increased PLQY of $1.9 \%$ with an absorption peak redshift of $8 \mathrm{~nm}$ (Fig. S9, ESI $\dagger$ ).

The alcohol-treated InP NC surface chemistry and oxidation are analysed by XPS. Fig. 5c shows the $\mathrm{P} 2 \mathrm{p}$ spectra while other elemental spectra are presented in Fig. S10 (ESI $\dagger$ ). The $\mathrm{P} 2 \mathrm{p}$ spectrum of the control sample clearly shows the existence of $\mathrm{P}-$ In and $\mathrm{P}-\mathrm{O}_{x}$ bonds as mentioned above. Similar $\mathrm{P} 2 \mathrm{p}$ peaks are observed in EtOH- and $\mathrm{MeOH}-\mathrm{InP}$ NCs. The $\mathrm{InPO}_{x} / \mathrm{InP}$ intensity ratios are 0.3, 2.3 and 2.7 for the control, $\mathrm{EtOH}-$ and $\mathrm{MeOH}-\mathrm{InP} \mathrm{NC}$ samples, respectively. Notably, a peak at $\sim 140 \mathrm{eV}$ ascribed to the $\mathrm{Zn}$ 3s signal in both the EtOH- and MeOH-InP samples is observed.

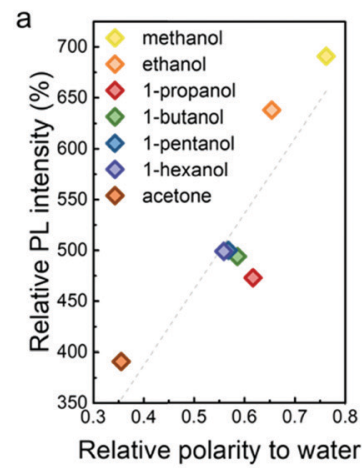

d

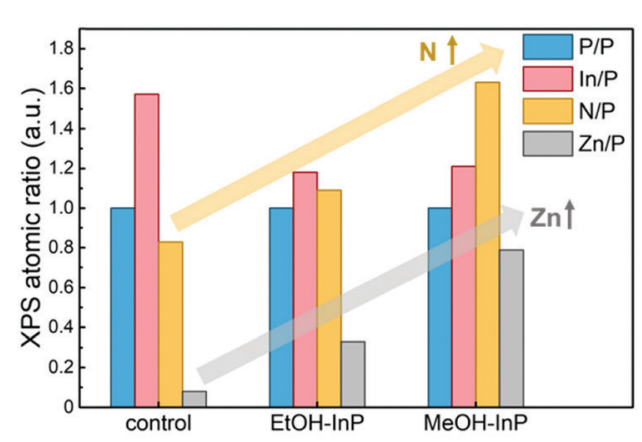

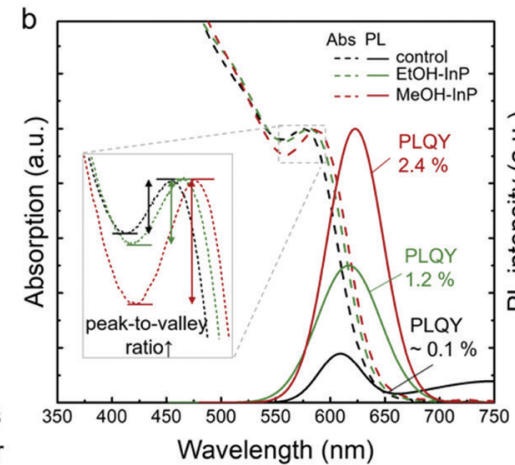

e

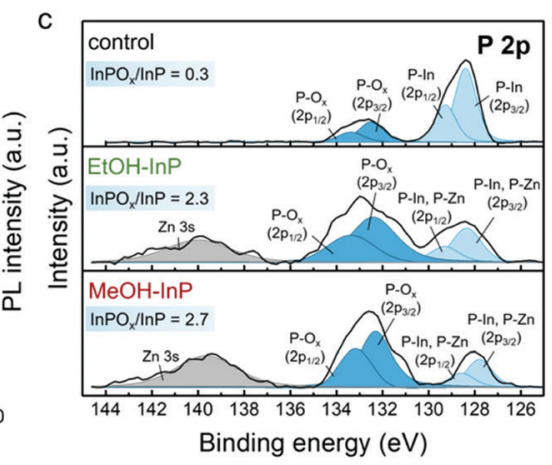

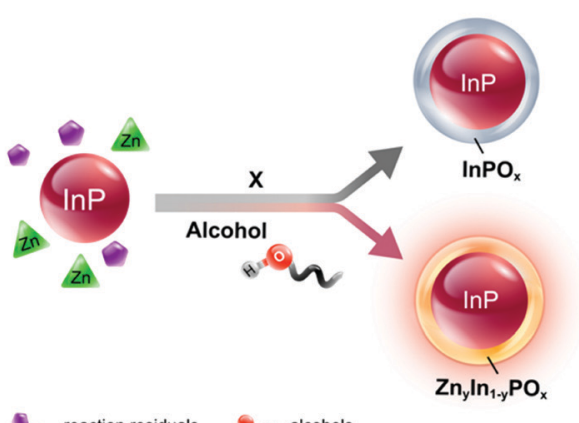

Fig. 5 Alcohol treatment of InP NCs. (a) The relation between alcohol polarity and the corresponding PL intensity after the aging process. (b) PL and optical absorption spectra of the control, ethanol-treated (EtOH-) and methanol-treated ( $\mathrm{MeOH}-)$ InP NC samples. The control sample is prepared by treating InP NCs directly with acetone. The inset shows the definition of peak-to-valley ratio in the absorption curves. (c) XPS P 2p spectra of InP NCs. The oxidized InP leads to complicated $\mathrm{P}-\mathrm{O}_{x}$ binding (e.g., InPO $\mathrm{P}_{4}$ and $\mathrm{P}_{2} \mathrm{O}_{5}$ ) and thus a broad and ambiguous peak at around $133 \mathrm{eV}$. (d) XPS In/P, N/P and $\mathrm{Zn} / \mathrm{P}$ atomic ratios of the control, EtOH- and $\mathrm{MeOH}-\mathrm{InP} \mathrm{NC}$ samples. The atomic ratio of $\mathrm{P}$ is normalized to 1 while other elements are shown in a relative atomic ratio to $\mathrm{P}$. (e) A sketch illustrates the formation of $I n \mathrm{PO}_{x}$ over $\mathrm{InP} N C s$ in a general aging process, and the formation of a $\mathrm{Zn}_{y} / \mathrm{n}_{1-y} P \mathrm{PO}_{x}$ layer either by adding alcohols during aging or the alcohol treatment. 
Atomic ratios of $\mathrm{Zn} / \mathrm{P}, \mathrm{In} / \mathrm{P}$ and $\mathrm{N} / \mathrm{P}$ are shown in Fig. $5 \mathrm{~d}$. The $\mathrm{Zn} / \mathrm{P}$ ratios are approximately $0.1,0.3$ and 0.8 for the control, $\mathrm{EtOH}-$ and $\mathrm{MeOH}-\mathrm{InP} \mathrm{NC}$ samples, respectively. In the $\mathrm{Zn} 2 \mathrm{p}_{3 / 2}$ spectra for both the EtOH- and $\mathrm{MeOH}-\mathrm{InP}$ NC samples, increased $\mathrm{Zn}-\mathrm{P}$ and $\mathrm{Zn}-\mathrm{O}$ bonds are found (Fig. S10, ESI $\dagger$ ). ${ }^{60,61}$ In addition, the EtOHand $\mathrm{MeOH}-\mathrm{InP}$ NC samples show decreased In/P ratios (control: 1.6, EtOH: 1.2 and $\mathrm{MeOH}$ : 1.2) along with increased $\mathrm{N} / \mathrm{P}$ ratios (control: 0.8, EtOH: 1.1 and $\mathrm{MeOH}: 1.6)$.

The intentionally added alcohols and the post alcohol treatment would play a similar role in the surface chemistry of the InP NCs because of their similar PL enhancement and absorption peak redshift. The higher polarity methanol may have a stronger interaction with InP NCs or reaction residuals/excess ligand to induce surface passivation of NCs with higher PL intensity and larger redshift in spectral peaks. An increase in peak-to-valley ratio in the absorption curve is an indirect indication of the better quality or narrower size distribution of the EtOH- and MeOH-InP NCs. ${ }^{22}$ Furthermore, the immiscibility between EG and InP/toluene indicates that a rapid reaction between high polarity alcohol and InP NCs occurs, which only needs a short reaction time by simply stirring to induce PL enhancement and a spectral peak redshift.

On the basis of increased $\mathrm{P}-\mathrm{O}_{x}$ oxidation, lifted $\mathrm{Zn}$ content and $\mathrm{Zn}-\mathrm{O} / \mathrm{Zn}-\mathrm{P}$ existence in alcohol-treated $\mathrm{InP} \mathrm{NCs}$, it is suggested that a mixed $\mathrm{Zn}_{y} \mathrm{In}_{1-y} \mathrm{PO}_{x}$ passivation layer should form at the InP NC surface. When alcohol exists in an OAmcapped InP NC solution, the InP surface oxidation may occur because of the interaction of InP NCs with alcohol. The alcohol may trigger $\mathrm{Zn}-\mathrm{P} / \mathrm{Zn}-\mathrm{O}$ reactions and form the mixed $\mathrm{Zn}_{y} \mathrm{In}_{1-y} \mathrm{PO}_{x}$ passivation layer. $\mathrm{P}-\mathrm{Zn}$ binding in the $\mathrm{P} 2 \mathrm{p}$ spectra may also be observed, but the P-Zn highly overlap with P-In and thus become indistinguishable. ${ }^{62,63}$ The slight decrease in In/P ratio could be a result of partial $\mathrm{Zn}^{2+}$ substitution for surface $\mathrm{In}^{3+}$ on the InP NCs during the formation of $\mathrm{Zn}_{y} \mathrm{In}_{1-y} \mathrm{PO}_{x}$. The redshift in the spectral peaks may be ascribed to increase of $\mathrm{N} / \mathrm{P}$ ratio with an additional OAm ligand on the
NC surface. Formation of $\mathrm{Zn}_{y} \mathrm{In}_{1-y} \mathrm{PO}_{x}$ with a similar band alignment as the InP/ZnO type II core/shell structure may also contribute to redshifted spectral peaks. ${ }^{64}$ It is concluded that alcohol induces more incorporation of not only $\mathrm{Zn}^{2+}$ but also OAm ligand on the NC surface. Alcohol itself directly binding to the InP NC surface is excluded since the binding of alcohols would lead to decreases in the OAm ligands and N/P ratio. It is proposed that when protic alcohols are added into aprotic organic solvents (i.e., toluene and OAm), preferential solvation may occur to the unfavorable impurities on the NC surface and thereby be carried off from the NC surface. ${ }^{65}$ At the time, $\mathrm{Zn}^{2+}$ and OAm in the solution along with trace water and oxygen are induced by alcohols to react with InP NCs and form a mixed $\mathrm{Zn}_{y} \mathrm{In}_{1-y} \mathrm{PO}_{x}$ oxide layer, leading to surface passivation with improved PL intensity and redshifted spectral peaks. Increase in PL lifetime of both the EtOH- and $\mathrm{MeOH}-\mathrm{InP}$ NC samples also suggests the surface passivation effect from the $\mathrm{Zn}_{y} \mathrm{In}_{1-y} \mathrm{PO}_{x}$ oxide layer (Fig. S11 and Table S6, ESI $\dagger$ ).

In a general aging process without any additives, the InP NCs show the formation of an $\operatorname{InPO}_{x}$ passivation layer with an increased PL intensity and blue-shifted absorption peak. However, if alcohol is introduced as an additive in the aging process or InP NCs are directly treated with alcohols, a mixed $\mathrm{Zn}_{y} \mathrm{In}_{1-y} \mathrm{PO}_{x}$ would form over the NC surface with increased PL intensity and redshifted spectral peaks (Fig. 5e).

The material stability of alcohol-treated InP NCs is examined by their PL intensity and absorption peak shift in the aging process. The control, EtOH- and $\mathrm{MeOH}-\mathrm{InP}$ NC samples are aged in light/air for $168 \mathrm{~h}$. As displayed in Fig. 6a, the control sample shows a large decrease in PL intensity (>90\%) and a large absorption peak blueshift $(\sim 60 \mathrm{~nm})$ after $168 \mathrm{~h}$ aging. The EtOH- and $\mathrm{MeOH}-\mathrm{InP}$ NC samples show increased PL intensities (330-370\%) and rather small absorption peak blueshifts (12-16 nm) after $168 \mathrm{~h}$ aging. Feasibility of alcohol treatment to aminophosphine-based InP NCs is also demonstrated. By varying synthetic temperatures from 150 to $280{ }^{\circ} \mathrm{C}$, a

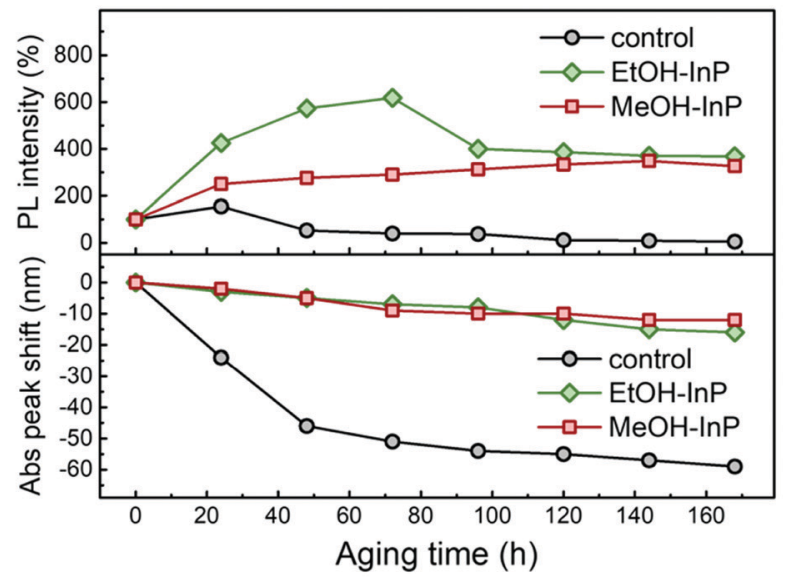

b

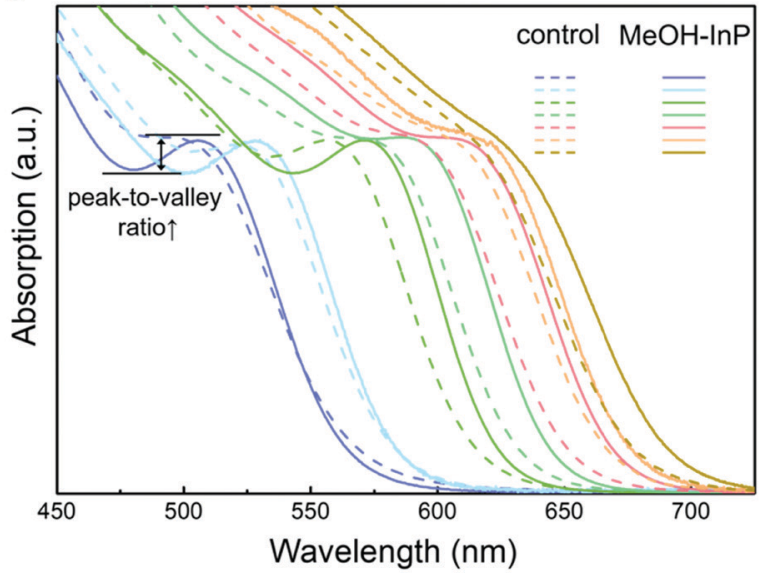

Fig. 6 (a) Photo-stability examined from PL intensity and absorption peak shift of the control, ethanol-and methanol-treated InP NC samples in a light/ air $168 \mathrm{~h}$ aging process. All three samples are in a concentration of $1 \mathrm{mM}$ with a total amount of $3 \mathrm{~mL}$ in toluene and aged in light/air for $168 \mathrm{~h}$. (b) Optical absorption spectra of InP NCs with absorption peaks spanning the green to red spectrum before and after methanol treatment. 
InP NCs with absorption peaks covering the green to red visible spectrum are prepared. All InP NCs are treated with methanol, and the resulting optical absorption spectra are shown in Fig. 6b. After methanol treatment, all InP NCs show redshifted absorption peaks and increased peak-to-valley ratios.

For the control sample, decreased PL intensity and blueshifted absorption peak suggest a sensitive nature and serious $\mathrm{InPO}_{x}$ oxidation of InP NCs after a light/air $168 \mathrm{~h}$ aging process. For the EtOH- and $\mathrm{MeOH}-\mathrm{InP}$ NC samples, the increased PL intensity and smaller blueshift in the absorption peak are attributed to better surface passivation from the $\mathrm{Zn}_{y} \mathrm{In}_{1-y} \mathrm{PO}_{x}$ oxide layer and ligand rearrangement over the NC surface as the aging time extends. ${ }^{53,66}$ The larger PL intensity at $72 \mathrm{~h}$ of aging of the EtOH-InP NCs is due to their lower original PLQY $(1.2 \%)$ and thus they have more room for enhancement. It is suggested that mixed $\mathrm{Zn}_{y} \mathrm{In}_{1-y} \mathrm{PO}_{x}$ has a better surface passivation effect than $\operatorname{InPO}_{x}$ on the InP NCs. The feasibility of surface passivation by alcohol treatment is supported by the InP NCs with various absorption peaks. The alcohol treatment with the formation of a mixed $\mathrm{Zn}_{y} \mathrm{In}_{1-y} \mathrm{PO}_{x}$ layer, more specifically, not only improves the PL instantly but also enhances the photostability of NCs against aging. It is proposed that adding methanol into aminophosphine-based InP NCs could prevent InP NCs from degradation for long-time storage and may be beneficial for subsequent overcoating and surface modification processes. These encouraging findings, either aging InP NCs with existing metal ions and alcohols or directly treating them with alcohols to trigger the formation of a $\mathrm{Zn}_{y} \mathrm{In}_{1-y} \mathrm{PO}_{x}$ passivation layer, highlight the special surface chemistry of aminophosphine-based InP NCs.

\section{Conclusions}

The effect of existing metal ions and intentionally added alcohols on aminophosphine-based InP NC oxidation, PL and photo-stability are investigated. In a general aging process without any additive, InP NCs show an enhanced InPO $_{x}$ oxide layer with increased PL intensity and blue-shifted absorption peak. The existing reaction residuals (i.e., $\mathrm{In}^{3+}, \mathrm{Zn}^{2+}, \mathrm{Cl}^{-}$, $\mathrm{P}(\mathrm{NHR})_{4}{ }^{+}$and $\left.\mathrm{OAm}\right)$ can alleviate InP NC oxidation in the aging process and are not involved in the $\mathrm{InPO}_{x}$ layer. With intentionally added alcohols, InP NCs with reaction residuals result in increased PL intensity with redshifted spectral peaks. It is found that alcohol triggers the coupling of $\mathrm{Zn}^{2+}$ from reaction residuals to form a mixed $\mathrm{Zn}_{y} \mathrm{In}_{1-y} \mathrm{PO}_{x}$ oxide passivation layer on an alcohol-treated InP NC surface. The alcohol-treated InP NCs demonstrate improved PLQY ( $\sim 25$ folds) and photostability ( $\sim 9$ folds) against aging. This work demonstrates a new insight to surface chemistry and surface modification for aminophosphine-based InP NCs.

\section{Conflicts of interest}

The authors declare no conflict of interest.

\section{Acknowledgements}

This work was financially supported by the Ministry of Science and Technology (Taiwan) (contract no: 104-2623-E-007-007-ET and 105-2119-M-007-031). The authors gratefully acknowledge Yen-Ting Liao (Instrumentation Centre at National Tsing-Hua University) for the TRPL analysis.

\section{References}

1 D. V. Talapin, J. S. Lee, M. V. Kovalenko and E. V. Shevchenko, Chem. Rev., 2010, 110, 389-458.

2 D. J. Norris and M. G. Bawendi, Phys. Rev. B: Condens. Matter Mater. Phys., 1996, 53, 16338-16346.

3 Y. Shirasaki, G. J. Supran, M. G. Bawendi and V. Bulovic, Nat. Photonics, 2013, 7, 13-23.

4 B. S. Mashford, M. Stevenson, Z. Popovic, C. Hamilton, Z. Q. Zhou, C. Breen, J. Steckel, V. Bulovic, M. Bawendi, S. Coe-Sullivan and P. T. Kazlas, Nat. Photonics, 2013, 7, 407-412.

5 S. J. Ho, H. C. Hsu, C. W. Yeh and H. S. Chen, ACS Appl. Mater. Interfaces, 2020, 12, 33346-33351.

6 S. J. Ho and H. S. Chen, J. Mater. Chem. C, 2020, 8, 4309-4313.

7 G. H. Chen, C. W. Yeh, M. H. Yeh, S. J. Ho and H. S. Chen, Chem. Commun., 2015, 51, 14750-14753.

8 I. L. Medintz, A. R. Clapp, H. Mattoussi, E. R. Goldman, B. Fisher and J. M. Mauro, Nat. Mater., 2003, 2, 630-638.

9 F. Pinaud, X. Michalet, L. A. Bentolila, J. M. Tsay, S. Doose, J. J. Li, G. Iyer and S. Weiss, Biomaterials, 2006, 27, 1679-1687.

10 V. I. Klimov, A. A. Mikhailovsky, S. Xu, A. Malko, J. A. Hollingsworth, C. A. Leatherdale, H. J. Eisler and M. G. Bawendi, Science, 2000, 290, 314-317.

11 V. I. Klimov, S. A. Ivanov, J. Nanda, M. Achermann, I. Bezel, J. A. McGuire and A. Piryatinski, Nature, 2007, 447, 441-446.

12 N. Yaacobi-Gross, M. Soreni-Harari, M. Zimin, S. Kababya, A. Schmidt and N. Tessler, Nat. Mater., 2011, 10, 974-979.

13 S. A. McDonald, G. Konstantatos, S. G. Zhang, P. W. Cyr, E. J. D. Klem, L. Levina and E. H. Sargent, Nat. Mater., 2005, 4, 138-142.

14 D. Battaglia and X. G. Peng, Nano Lett., 2002, 2, 1027-1030. 15 L. Li and P. Reiss, J. Am. Chem. Soc., 2008, 130, 11588-11589. 16 C. C. Hung, S. J. Ho, C. W. Yeh, G. H. Chen, J. H. Huang and H. S. Chen, J. Phys. Chem. C, 2017, 121, 28373-28384.

17 E. M. Janke, N. E. Williams, C. X. She, D. Zherebetskyy, M. H. Hudson, L. L. Wang, D. J. Gosztola, R. D. Schaller, B. Lee, C. J. Sun, G. S. Engel and D. V. Talapin, J. Am. Chem. Soc., 2018, 140, 15791-15803.

18 A. Tarantini, K. D. Wegner, F. Dussert, G. Sarret, D. Beal, L. Mattera, C. Lincheneau, O. Proux, D. Truffier-Boutry, C. Moriscot, B. Gallet, P. H. Jouneau, P. Reiss and M. Carriere, Nanoimpact, 2019, 14, 100168.

19 T. G. Kim, D. Zherebetskyy, Y. Bekenstein, M. H. Oh, L. W. Wang, E. Jang and A. P. Alivisatos, ACS Nano, 2018, 12, 11529-11540.

20 W. S. Song, H. S. Lee, J. C. Lee, D. S. Jang, Y. Choi, M. Choi and H. Yang, J. Nanopart. Res., 2013, 15, 1750. 
21 M. D. Tessier, D. Dupont, K. De Nolf, J. De Roo and Z. Hens, Chem. Mater., 2015, 27, 4893-4898.

22 J. H. Jo, D. Y. Jo, S. H. Lee, S. Y. Yoon, H. B. Lim, B. J. Lee, Y. R. Do and H. Yang, ACS Appl. Nano Mater., 2020, 3, 1972-1980.

23 E. Cho, T. Kim, S. M. Choi, H. Jang, K. Ming and E. Jang, ACS Appl. Nano Mater., 2018, 1, 7106-7114.

24 M. D. Tessier, E. A. Baquero, D. Dupont, V. Grigel, E. Bladt, S. Bals, Y. Coppel, Z. Hens, C. Nayral and F. Delpech, Chem. Mater., 2018, 30, 6877-6883.

25 S. Tamang, C. Lincheneau, Y. Hermans, S. Jeong and P. Reiss, Chem. Mater., 2016, 28, 2491-2506.

26 J. L. Stein, W. M. Holden, A. Venkatesh, M. E. Mundy, A. J. Rossini, G. T. Seidler and B. M. Cossairt, Chem. Mater., 2018, 30, 6377-6388.

27 K. E. Hughes, J. L. Stein, M. R. Friedfeld, B. M. Cossairt and D. R. Gamelin, ACS Nano, 2019, 13, 14198-14207.

28 L. S. Xie, D. K. Harris, M. G. Bawendi and K. F. Jensen, Chem. Mater., 2015, 27, 5058-5063.

29 S. C. Huang, C. W. Yeh, G. H. Chen, M. C. Liu and H. S. Chen, ACS Appl. Mater. Interfaces, 2019, 11, 2516-2525.

30 H. Y. Liu, Q. Dong and R. Lopez, Nanomaterials, 2018, 8, 341.

31 A. Y. Nazzal, X. Y. Wang, L. H. Qu, W. Yu, Y. J. Wang, X. G. Peng and M. Xiao, J. Phys. Chem. B, 2004, 108, 5507-5515.

32 D. Q. Chen, Y. Liu, C. B. Yang, J. S. Zhong, S. Zhou, J. K. Chen and H. Huang, Nanoscale, 2019, 11, 17216-17221.

33 C.-W. Yeh, G.-H. Chen, S.-J. Ho and H.-S. Chen, ACS Appl. Nano Mater., 2019, 2, 5290-5301.

34 F. Liu, C. Ding, Y. H. Zhang, T. Karnisaka, Q. Zhao, J. M. Luther, T. Toyoda, S. Hayase, T. Minemoto, K. Yoshino, B. Zhang, S. Dai, J. K. Jiang, S. X. Tao and Q. Shen, Chem. Mater., 2019, 31, 798-807.

35 H. S. Chen and R. V. Kumar, Cryst. Growth Des., 2009, 9, 4235-4238.

36 M. H. Yeh, P. H. Chen, Y. C. Yang, G. H. Chen and H. S. Chen, ACS Appl. Mater. Interfaces, 2017, 9, 10788-10797.

37 A. Cros-Gagneux, F. Delpech, C. Nayral, A. Cornejo, Y. Coppel and B. Chaudret, J. Am. Chem. Soc., 2010, 132, 18147-18157.

38 H. Virieux, M. Le Troedec, A. Cros-Gagneux, W. S. Ojo, F. Delpech, C. Nayral, H. Martinez and B. Chaudret, J. Am. Chem. Soc., 2012, 134, 19701-19708.

39 P. Ramasamy, B. Kim, M. S. Lee and J. S. Lee, Nanoscale, 2016, 8, 17159-17168.

40 Y. H. Won, O. Cho, T. Kim, D. Y. Chung, T. Kim, H. Chung, H. Jang, J. Lee, D. Kim and E. Jang, Nature, 2019, 575, 634-638.

41 A. Vikram, A. Zahid, S. S. Bhargava, L. P. Keating, A. Sutrisno, A. Khare, P. Trefonas, M. Shim and P. J. A. Kenis, Chem. Mater., 2020, 32, 3577-3584.

42 O. I. Micic, J. Sprague, Z. H. Lu and A. J. Nozik, Appl. Phys. Lett., 1996, 68, 3150-3152.

43 H. X. Fu and A. Zunger, Phys. Rev. B: Condens. Matter Mater. Phys., 1997, 56, 1496-1508.
44 P. R. Chen, K. Y. Lai, C. W. Yeh and H. S. Chen, ACS Appl. Nano Mater., 2021, 4, 3977-3988.

45 A. Buffard, S. Dreyfuss, B. Nadal, H. Heuclin, X. Z. Xu, G. Patriarche, N. Mezailles and B. Dubertret, Chem. Mater., 2016, 28, 5925-5934.

46 Y. Zhang, J. He, P. N. Wang, J. Y. Chen, Z. J. Lu, D. R. Lu, J. Guo, C. C. Wang and W. L. Yang, J. Am. Chem. Soc., 2006, 128, 13396-13401.

47 L. P. Liu, Q. Peng and Y. D. Li, Inorg. Chem., 2008, 47, 3182-3187.

48 A. V. Baranov, Y. P. Rakovich, J. F. Donegan, T. S. Perova, R. A. Moore, D. V. Talapin, A. L. Rogach, Y. Masumoto and I. Nabiev, Phys. Rev. B: Condens. Matter Mater. Phys., 2003, 68, 165306.

49 H. S. Chen, B. Lo and J. Y. Huang, RSC Adv., 2014, 4, 2614-2619.

50 D. A. Hines, M. A. Becker and P. V. Kamat, J. Phys. Chem. C, 2012, 116, 13452-13457.

51 G. V. Shcherbatyuk, R. H. Inman and S. Ghosh, J. Appl. Phys., 2011, 110, 053518.

52 V. Krivenkov, P. Samokhvalov, M. Zvaigzne, I. Martynov, A. Chistyakov and I. Nabiev, J. Phys. Chem. C, 2018, 122, 15761-15771.

53 C. Carrillo-Carrion, S. Cardenas, B. M. Simonet and M. Valcarcel, Chem. Commun., 2009, 5214-5226.

54 E. A. Baquero, H. Virieux, R. A. Swain, A. Gillet, A. CrosGagneux, Y. Coppel, B. Chaudret, C. Nayral and F. Delpech, Chem. Mater., 2017, 29, 9623-9627.

55 T. Chasse, R. Franke, C. Urban, R. Franzheld, P. Streubel and A. Meisel, J. Electron Spectrosc. Relat. Phenom., 1993, 62, 287-308.

56 M. Faur, M. Faur, D. T. Jayne, M. Goradia and C. Goradia, Surf. Interface Anal., 1990, 15, 641-650.

57 S. Jeong, M. Achermann, J. Nanda, S. Lvanov, V. I. Klimov and J. A. Hollingsworth, J. Am. Chem. Soc., 2005, 127, 10126-10127.

58 J. K. Cooper, A. M. Franco, S. Gul, C. Corrado and J. Z. Zhang, Langmuir, 2011, 27, 8486-8493.

59 C. Reichardt and T. Welton, Solvents and solvent effects in organic chemistry, John Wiley \& Sons, 2011.

60 R. K. Brow, J. Non-Cryst. Solids, 1996, 194, 267-273.

61 U. Elrod, M. C. Luxsteiner, M. Obergfell, E. Bucher and L. Schlapbach, Appl. Phys. B, 1987, 43, 197-201.

62 E. J. Luber, M. H. Mobarok and J. M. Buriak, ACS Nano, 2013, 7, 8136-8146.

63 G. M. Kimball, J. P. Bosco, A. M. Muller, S. F. Tajdar, B. S. Brunschwig, H. A. Atwater and N. S. Lewis, J. Appl. Phys., 2012, 112, 106101.

64 H. B. Jalali, M. M. Aria, U. M. Dikbas, S. Sadeghi, B. G. Kumar, M. Sahin, I. H. Kavakli, C. W. Ow-Yang and S. Nizamoglu, ACS Nano, 2018, 12, 8104-8114.

65 T. W. Bentley, D. N. Ebdon, E. J. Kim and I. S. Koo, J. Org. Chem., 2005, 70, 1647-1653.

66 H. Asami, Y. Abe, T. Ohtsu, I. Kamiya and M. Hara, J. Phys. Chem. B, 2003, 107, 12566-12568. 